\title{
IncRNA GAS8-AS1 regulates cancer cell proliferation and predicts poor survival of patients with gastric cancer
}

\author{
CHAO LI, HUI WANG, SONG MENG, JIAN HONG, \\ LIBIN YAO, YONG SHAO and XIAOCHENG ZHU

\begin{abstract}
Department of General Surgery, The Affiliated Hospital of Xuzhou Medical University, Xuzhou, Jiangsu 221006, P.R. China
\end{abstract}

Received December 19, 2020; Accepted September 22, 2021

DOI: $10.3892 / \mathrm{ol} .2021 .13166$

\begin{abstract}
Long non-coding (lnc)RNAs have been recognized as important regulators in gastric cancer. IncRNA GAS8-AS1 is considered a tumor suppressor in multiple types of cancer, such as papillary thyroid carcinoma, ovarian cancer and colorectal cancer. However, the specific role of GAS8-AS1 in gastric cancer remains to be fully elucidated. The aim of the present study was to investigate the role of GAS8-AS1 in gastric cancer and its potential underlying mechanisms of action. The expression levels of GAS8-AS1, microRNA (miR)-21-3p, PTEN and pyruvate dehydrogenase (E1) alpha subunit gene (PDHA1) in gastric cancer and non-cancerous tissues, as well as in gastric cancer cell lines, were detected using reverse transcription-quantitative PCR. Cell proliferation was detected by using a Cell Counting Kit-8 assay. Cell migration and invasion were detected using a Transwell assay. Results of the present study demonstrated that the expression levels of GAS8-AS1 in gastric cancer tissues were significantly decreased, whereas its expression did not differ among cancer tissues at different clinical stages. Low expression levels of GAS8-AS1 predicted poor 5-year survival rates for 70 patients with gastric adenocarcinoma from the Affiliated Hospital of Xuzhou Medical University (Xuzhou, China) during patient follow-up. In addition, the expression levels of miR-21-3p were markedly increased in cancer tissues, and miR-21-3p expression was negatively associated with the expression of GAS8-AS1. The direct interaction between GAS8-AS1 and miR-21-3p was predicted using the starBase database and was confirmed by using an RNA pull-down assay. In gastric cell lines, the overexpression of GAS8-AS1 reduced the expression levels of mature miR-21-3p but did not affect the expression of miR-21-3p precursor, while
\end{abstract}

Correspondence to: Dr Xiaocheng Zhu, Department of General Surgery, The Affiliated Hospital of Xuzhou Medical University, 99 Huaihai West Road, Xuzhou, Jiangsu 221006, P.R. China E-mail: re95523@163.com

Key words: gastric cancer, long non-coding RNA GAS8-AS1, microRNA-21-3p, prognosis the overexpression of miR-21-3p did not, in turn, affect the expression of GAS8-AS1. In addition, the overexpression of GAS8-AS1 inhibited cancer cell proliferation, while the overexpression of miR-21-3p promoted cancer cell proliferation and attenuated the effects of GAS8-AS1. Overexpression of miR-21-3p promoted cancer cell migration and invasion, whereas overexpression of GAS8-AS1 did not affect cell migration or invasion. In summary, results of the present study have demonstrated that GAS8-AS1 acts as a tumor suppressor in gastric cancer, and it may inhibit cancer cell proliferation by downregulating miR-21-3p.

\section{Introduction}

Gastric cancer is a frequently diagnosed cancer worldwide, with the highest incidence rates in regions such as Central and South America, Eastern Europe and East Asia (1). Early-stage gastric cancer is radically treated with surgery; however, effective therapies for advanced gastric cancer are still lacking (2). A considerable number of tumor suppressors and oncogenes have been identified as critical players in gastric cancer. For instance, microRNA-223 (miRNA/miR-223) and long non-coding RNA (lncRNA) UCA1 have been found to have oncogenic roles in gastric cancer $(3,4)$, while miR-4317 and lncRNA MEG3 display anticancer functions in gastric cancer $(5,6)$. However, the pathogenesis of this disease remains to be fully elucidated (7), leading to difficulties in clinical treatment.

The functions of lncRNAs, which are $>200$ nucleotides in length, in cancer occurrence and progression, such as carcinogenesis, metastasis and chemoresistance have recently been investigated (8). In effect, regulation of the expression of specific lncRNAs has demonstrated potential in cancer treatment and prevention $(9,10)$. Various lncRNAs have been characterized as critical regulators in gastric cancer. For example, IncRNA H19, UCA1 and HOXA11-AS have been found to enhance the carcinogenesis and metastasis of gastric cancer $(4,11,12)$, while IncRNA MEG3 and MIR22HG have been found to inhibit gastric cancer progression $(6,13)$. Recent studies have reported that lncRNA GAS8-AS1 (chromosome 16) is a tumor suppressor in multiple types of cancer. For example, GAS8-AS1 has demonstrated inhibitory effects during cell proliferation in papillary thyroid carcinoma (14), cancer cell migration and invasion in osteosarcoma (15), and 
cancer cell proliferation in glioblastoma (16). Mechanistically, GAS8-AS1 may function by regulating downstream miRNAs and target genes, such as the miR-187-3p/autophagy protein 5 axis and the miR-135b-5p/G1/S-specific cycin-D2 axis $(14,17)$. However, the role of GAS8-AS1 in gastric cancer remains to be fully elucidated.

miR-21-3p has been identified as an oncogenic miRNA and is frequently upregulated in a variety of malignancies such as colorectal and breast cancer, with roles in multiple processes of cancer development, such as cancer cell proliferation, migration and invasion $(18,19)$. Previous studies have revealed that miR-21-3p plays a pivotal role in the pathogenesis and progression of gastric cancer $(20,21)$. Therefore, the present study aimed to investigate the role of GAS8-AS1 in gastric cancer and determine whether its effects are mediated by regulating miR-21-3p. A total of 70 patients with gastric adenocarcinoma were involved in the study. GAS8-AS1 and miR-21-3p levels in cancer and non-cancerous tissues were detected, and a survival analysis was performed. In gastric cell lines, RNA pull-down and reverse transcription-quantitative (RT-q)PCR assays were performed to explore the interaction between GAS8-AS1 and miR-21-3p. Finally, CCK-8 and Transwell assays were performed to assess the effects of GAS8-AS1 and miR-21-3p on cell proliferation, migration and invasion.

\section{Materials and methods}

Patients. The present study included 70 patients with gastric adenocarcinoma (40 male and 30 female patients; age, 48.4 \pm 7.1 years; range, 30-66 years) selected from 168 patients who were admitted at the Affiliated Hospital of Xuzhou Medical University (Xuzhou, China) between March 2010 and March 2013.

The inclusion criteria were as follows: i) First-time diagnosis; ii) no previous history of cancer; and iii) completed 5 -year follow-up after admission. The exclusion criteria were as follows: i) Diagnosis of other clinical conditions; and ii) patients who failed to complete the 5-year follow-up. There were 12, 18, 22 and 18 cases with American Joint Committee on Cancer clinical stage I, II, III and IV, respectively (22). These 70 patients included 43 cases of high-grade (poorly differentiated) and 27 cases of low-grade (highly differentiated) cancer.

All the patients provided written informed consent before involved in the present study. The present study was approved by the Ethics Committee of the Affiliated Hospital of Xuzhou Medical University prior to patient admission (approval no. XZNU-2010-032).

Tissues and cells. Gastric cancer and non-cancerous adjacent tissues were collected through gastric biopsies. SNU-1 and AGS (American Type Culture Collection) gastric cancer cell lines and a normal gastric cell line Hs 738.St/Int (American Type Culture Collection) were used. The cells were authenticated using the STR method and mycoplasma contamination was excluded using the MycAway ${ }^{\text {TM }}$-Color One-Step Mycoplasma Detection kit (cat. no. 40611ES25; Shanghai Yeasen Biotechnology Co., Ltd.). Cells were cultivated in 90\% RPMI-1640 medium (Invitrogen; Thermo Fischer Scientific, Inc.) supplemented with 10\% FBS (Thermo Fisher Scientific, Inc.) at $37^{\circ} \mathrm{C}$ for $72 \mathrm{~h}$ before the experiments.
Follow-up. From the day of admission, all 70 patients were followed up monthly using telephone communication for 5 years to record survival rates.

$R T-q P C R$. Total RNA from the cancer and non-cancerous adjacent tissues, as well as from the SNU-1, AGS and Hs 738 . $\mathrm{St} /$ Int cell lines, was extracted using RNAzol ${ }^{\circledR}$ reagent (Sigma-Aldrich; Merck KGaA). Total RNA was reverse transcribed using SuperScript ${ }^{\mathrm{TH}}$ IV First-Strand Synthesis System (Invitrogen; Thermo Fisher Scientific, Inc.) to synthesize cDNA according to the manufacturer's protocol. PowerUp $^{\text {TI }}$ SYBR $^{\text {TI }}$ Green Master Mix (Applied Biosystems; Thermo Fisher Scientific, Inc.) was used for the preparation of qPCR reactions. The expression levels of GAS8-AS1 were determined with $18 \mathrm{~S}$ rRNA as the endogenous control. To measure the expression of miR-21-3p, miRNA extraction was performed using miRNeasy kit (Qiagen, Inc.), and U6 was used as the internal control of miR-21-3p. The All-in-One ${ }^{\mathrm{Tw}}$ miRNA qRT-PCR Detection kit (GeneCopoeia, Inc.) was used to perform RT-qPCR. The PCR thermal cycling conditions were as follows: $95^{\circ} \mathrm{C}$ for $1 \mathrm{~min}$, followed by 40 cycles of $95^{\circ} \mathrm{C}$ for $15 \mathrm{sec}, 58^{\circ} \mathrm{C}$ for $20 \mathrm{sec}$ and a final extension of $72^{\circ} \mathrm{C}$ for $45 \mathrm{sec}$. Cq values of target genes were normalized to internal controls using the $2^{-\Delta \Delta \mathrm{Cq}}$ method (23). The primer sequences are listed in Table I.

Transient transfection. Transient transfection was applied to investigate the effects of short-term overexpression of GAS8-AS1 and miR-21-3p on gastric cancer cell lines. The overexpression of GAS8-AS1 and miR-21-3p was achieved by transfecting $10 \mathrm{nM}$ GAS8-AS1 expression vector or miR-21-3p mimic into in vitro cultivated cells using Lipofectamine ${ }^{\circledR} 2000$ (Invitrogen; Thermo Fisher Scientific, Inc.) at room temperature for $24 \mathrm{~h}$. Backbone pcDNA3.1 vector (Invitrogen; Thermo Fisher Scientific, Inc.) was used to construct the expression vector of GAS8-AS1. Negative control (NC) miRNA (scrambled mimic; 5'-CCUUCCGAGAGAA GAGCC-3') and miR-21-3p mimic (5'-CGGGUAGCUGACC ACAAC-3') were purchased from Invitrogen (Thermo Fisher Scientific, Inc.). NC experiments were performed by transfecting cells with $10 \mathrm{nM}$ empty vector or NC miRNA. Control (C) cells were untransfected cells. The subsequent experiments were performed at $24 \mathrm{~h}$ post-transfection.

RNA pull-down assay. The interaction between GAS8-AS1 and miR-21-3p was determined by RNA pull-down assay using the Pierce Magnetic RNA-Protein Pull-Down kit (Thermo Fisher Scientific, Inc.) in accordance with the manufacturer's protocol. The desthiobiotin-labeled DNA probes targeting back-splicing sequence of miR-21-3p and NC miRNA, named Bio-miR-21-3p or Bio-NC, were purchased from Invitrogen; Thermo Fisher Scientific, Inc. As aforementioned, SNU-1 cells were co-transfected with $10 \mathrm{nM}$ GAS8-AS1 expression vector and $10 \mathrm{nM}$ Bio-miR-21-3p or Bio-NC at $37^{\circ} \mathrm{C}$ using Lipofectamine 2000. At $48 \mathrm{~h}$ post-transfection, $400 \mu \mathrm{l} \mathrm{TRIzol}^{\circledR}$ lysis buffer (Thermo Fisher Scientific, Inc.) was prepared and RNA pull-down was performed using $50 \mu \mathrm{l}$ streptavidin agarose magnetic beads (Thermo Fisher Scientific, Inc.) at room temperature. After incubation at $4^{\circ} \mathrm{C}$ for $1.5 \mathrm{~h}$ with rotation streptavidin magnetic beads were washed three times with 
Table I. Primers used for reverse transcription-quantitative PCR.

\begin{tabular}{|c|c|c|}
\hline Gene name & Forward, 5'-3' & Reverse, 5'-3' \\
\hline GAS8-AS1 & CAACGAGCAAACAAGAAGGA & TGAGCCAAACAGACCAGTCA \\
\hline PTEN & AAGACCATAACCCACCACAGC & CACCAGTTCGTCCCTTTCCA \\
\hline $18 S r R N A$ & CTACCACATCCAAGGAAGCA & TTTTTCGTCACTACCTCCCCG \\
\hline $\operatorname{miR}-21$ & GCCCGCTAGCTTATCAGACTGATG & GTGCAGGGTCCGAGGT \\
\hline U6 & ATTGGAACGATACAGAGAAGATT & GGAACGCTTCACGAATTTG \\
\hline
\end{tabular}

miR, microRNA.
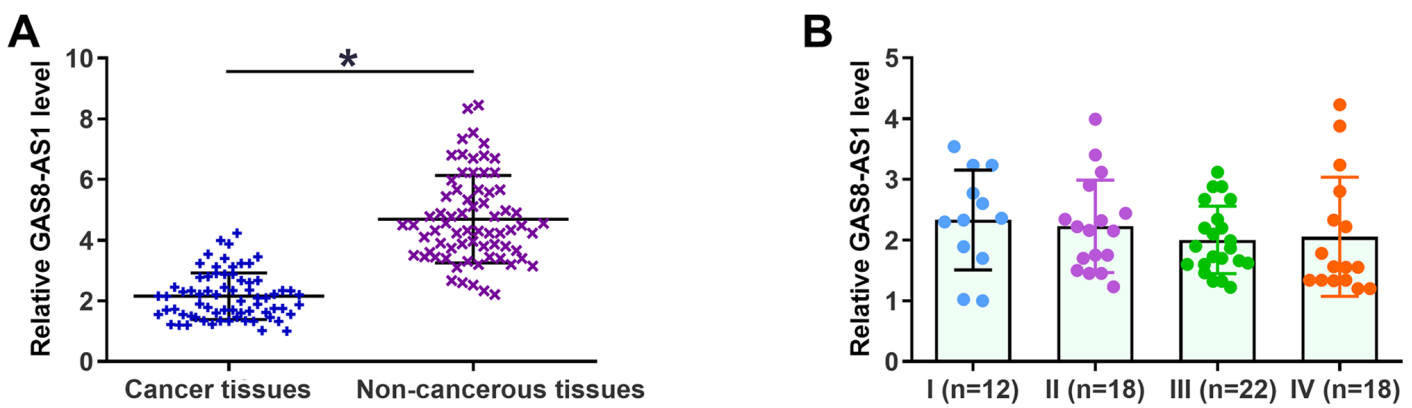

Figure 1. GAS8-AS1 is downregulated in gastric cancer tissues, but its expression is not affected by clinical stage. Reverse transcription-quantitative PCR results demonstrated that (A) the expression levels of GAS8-AS1 were significantly lower in cancer tissues compared with those in non-cancerous tissues, but (B) there were no significant differences in the expression levels of GAS8-AS1 among patients at different clinical stages. ${ }^{*} \mathrm{P}<0.05$.

the washing buffer from the Pierce Magnetic RNA-Protein Pull-Down kit, and then incubated with $50 \mu$ l Elution buffer at $37^{\circ} \mathrm{C}$ for $15 \mathrm{~min}$ with agitation. Afterwards, the mixture was centrifuged at $800 \mathrm{x} \mathrm{g}$ for $15 \mathrm{~min}$ at $4^{\circ} \mathrm{C}$. Total RNA was extracted from pull-down samples, which was followed by RT-qPCR, as aforementioned, to determine the expression of GAS8-AS1.

Cell Counting Kit-8 (CCK-8) assay. Cells were seeded into a 96-well plate $(5,000$ cells per well in $0.1 \mathrm{ml}$ medium) and the cells were cultured under the aforementioned conditions, followed by cell collection every $24 \mathrm{~h}$ until $96 \mathrm{~h}$. CCK-8 (Sigma-Aldrich; Merck KGaA) was added to reach $10 \%$ final concentration at $4 \mathrm{~h}$ before cell collection. Finally, OD values $(460 \mathrm{~nm})$ were measured.

Transwell assay. Cell migration and invasion were assessed by using Transwell assays. The migration assay was performed the same as the invasion assay but without the Matrigel precoating. For the invasion assay, the Transwell inserts were incubated with $100 \mu 1$ Matrigel (MilliporeSigma) at $37^{\circ} \mathrm{C}$ for $6 \mathrm{~h}$. Then, $1 \times 10^{5}$ cells were transferred into the upper chamber with $1 \mathrm{ml}$ serum-free RPMI-1640 medium, and $1 \mathrm{ml}$ RPMI-1640 medium containing $20 \%$ FBS was added into the lower chamber. After $48 \mathrm{~h}$ of culture at $37^{\circ} \mathrm{C}, 0.5 \%$ crystal violet (Sigma-Aldrich; Merck KGaA) was used to stain membranes for $20 \mathrm{~min}$ at room temperature. Finally, an optical microscope (Olympus Corporation) was used to observe the migratory cells (x200 magnification).

Statistical analysis. Each experiment was repeated three times to calculate the mean values, and the experimental data are presented as the mean \pm standard deviation. The statistical analysis was performed in SPSS ver. 22.0 (IBM Corp.). A paired Student's t-test was used to compare gastric cancer and non-cancerous tissues. One-way ANOVA followed by Tukey's post hoc test was used to compare different clinical stages or different cell treatment groups. Survival analysis was performed by dividing patients into low $(n=38)$ and high $(n=32)$ expression levels of GAS8-AS1 (cancer tissue) groups according to Youden's index (24). Kaplan-Meier plotter and log-rank test were used to plot and compare survival curves. Associations between clinical factors and the expression levels of GAS8-AS1 were analyzed using a $\chi 2$ test. Pearson's correlation coefficient analysis was performed to analyze the correlation between GAS8-AS1 level and miR-21-3p. StarBase Database (http://starbase.sysu.edu.cn/index.php) was used to predict the potential targets of GAS8-AS1. P $<0.05$ was considered to indicate a statistically significant difference.

\section{Results}

GAS8-AS1 is downregulated in patients with gastric cancer. The expression levels of GAS8-AS1 in gastric cancer and non-cancerous tissues were detected via RT-qPCR. As shown in Fig. 1A, the expression levels of GAS8-AS1 were significantly lower in gastric cancer tissues compared with those in non-cancerous tissues. However, the expression levels of GAS8-AS1 in cancerous tissues at different clinical stages revealed no significant difference $(\mathrm{P}>0.05$; Fig. $1 \mathrm{~B})$. The $\chi^{2}$ test revealed that the expression of GAS8-AS1 had no association with patients' age, sex, BMI, history of drinking and smoking, tumor grade or clinical stage (data not shown). 
A

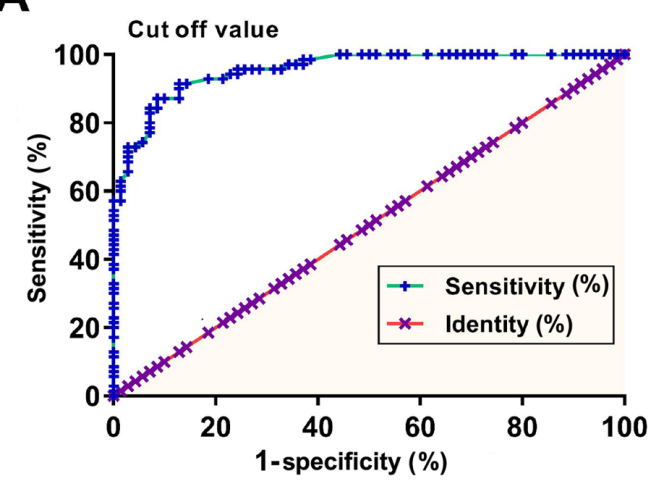

B

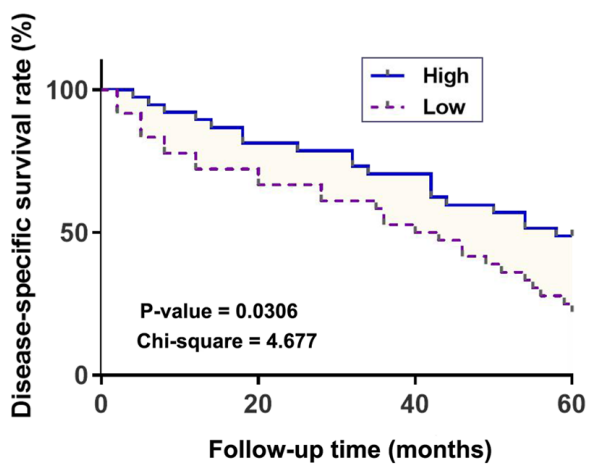

Figure 2. Lower expression levels of GAS8-AS1 in gastric cancer tissues predicts poor survival. (A) Patients were divided into high (n=32) and low ( $\mathrm{n}=38$ ) GAS8-AS1 expression (cancer tissue) groups according to Youden's index. (B) Survival curve analysis demonstrated that patients with low expression levels of GAS8-AS1 in cancer tissues exhibited a significantly lower disease-specific survival rate within the 5-year follow-up.

A

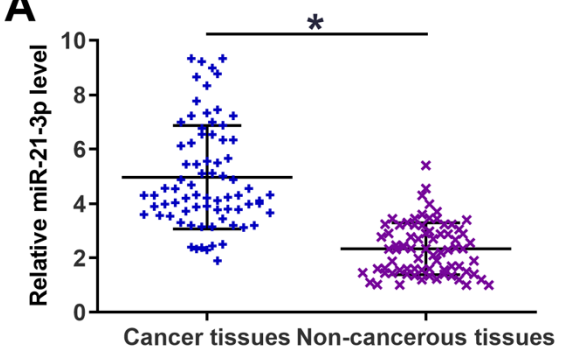

B

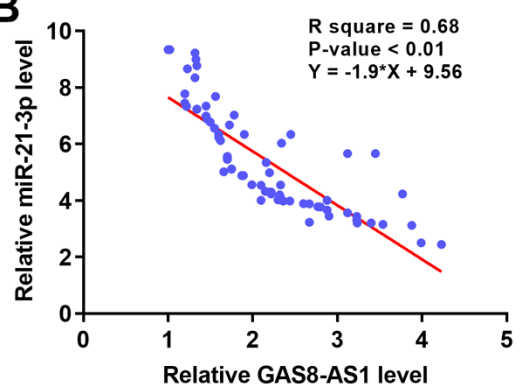

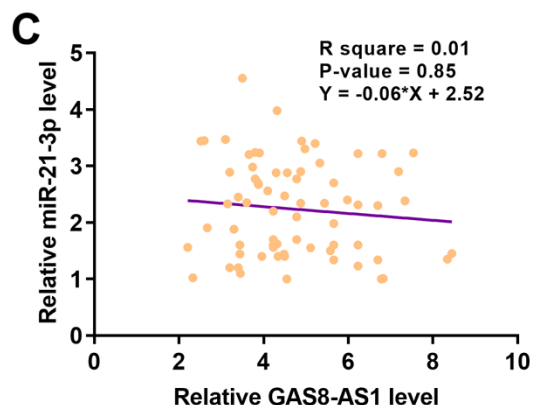

Figure 3. miR-21-3p is upregulated in gastric cancer tissues and its expression is inversely correlated with that of GAS8-AS1. Reverse transcription-quantitative PCR results demonstrated that (A) miR-21-3p was upregulated in gastric cancer tissues compared with non-cancerous tissues and its expression was inversely correlated with that of GAS8-AS1 in (B) gastric cancer tissues, but not in (C) adjacent non-cancerous tissues. ${ }^{\text {" }} \mathrm{P}<0.05$. miR, microRNA.

Reduced expression levels of GAS8-AS1 in gastric cancer tissues predict poor survival. Patients with gastric cancer were divided into high and low GAS8-AS1 expression groups according to Youden's index (Fig. 2A). Results of a survival curve analysis demonstrated that patients with low expression levels of GAS8-AS1 exhibited a markedly lower disease-specific survival rate within the 5-year follow-up compared to those with high expression levels (Fig. 2B).

miR-21-3p is upregulated in gastric cancer tissues and is inversely correlated with GAS8-AS1 expression. The expression levels of miR-21-3p in gastric cancer and non-cancerous tissues were also detected via RT-qPCR. As is evident in Fig. 3A $(\mathrm{P}<0.05)$, the expression levels of miR-21-3p in cancer tissues were significantly higher compared with those in adjacent non-cancerous tissues. Pearson's correlation coefficient analysis demonstrated that the expression levels of GAS8-AS1 and miR-21-3p were inversely correlated in cancer tissues, while they exhibited no correlation in the non-cancerous tissues (Fig. 3B and C).

Overexpression of GAS8-AS1 reduces the expression levels of miR-21-3p in gastric cancer cells. As shown in Fig. 4A, miR-21-3p was predicted to be a potential target of GAS8-AS1 using the starBase database. The RNA pull-down assay revealed that, compared with the Bio-NC pull-down sample, the Bio-miR-21-3p pull-down sample exhibited significantly higher expression levels of GAS8-AS1 (Fig. 4B; P<0.01), confirming the direct interaction between GAS8-AS1 and miR-21-3p. In addition, SNU-1 and AGS cells were transfected with GAS8-AS1 expression vector or miR-21-3p mimic and their corresponding controls.

As demonstrated in Fig. 5A, the expression level of GAS8-AS1 was significantly increased in cells transfected with the GAS8-AS1 expression vector compared with that in cells transfected with $\mathrm{NC}(\mathrm{P}<0.05)$. Overexpression of GAS8-AS1 significantly reduced the expression levels of miR-21-3p (Fig. 5B; $\mathrm{P}<0.05$ ) compared with the NC group, while the overexpression of miR-21-3p did not affect the expression of GAS8-AS1 (Fig. 5C and D; P<0.05) compared with the NC group. In addition, SNU-1 and AGS cells were also co-transfected with GAS8-AS1 expression vector and miR-21-3p mimic. The results demonstrated that the expression levels of GAS8-AS1 were significantly increased in the co-transfected cells compared with those in the NC group, while the expression levels of miR-21-3p in the co-transfected cells indicated no notable changes (Fig. 5E and F; $\mathrm{P}<0.05$ ) compared with those in the NC group. These results indicated that GAS8-AS1 directly targeted miR-21-3p and inhibited its expression.

GAS8-AS1 regulates cancer cell proliferation via miR-21-3p. Subsequently, the effects of GAS8-AS1 on the proliferation, migration and invasion of gastric cancer cells were investigated. 
A

GAS8-AS1 5'-...CAGCUACUGGGCA-3,
B

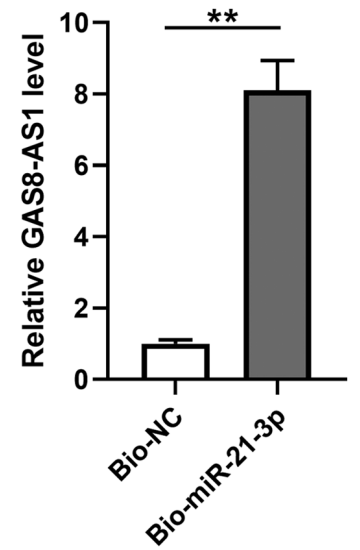

Figure 4. GAS8-AS1 reduces the expression of miR-21-3p by direct interaction. (A) Predicted binding site between GAS8-AS1 and miR-21-3p. (B) Bio-NC and Bio-miR-21-3p were used to pull down GAS8-AS1, followed by RNA isolation and reverse transcription-quantitative PCR to determine the expression of GAS8-AS1. ${ }^{* *} \mathrm{P}<0.01$. miR, microRNA; NC, negative control.

A

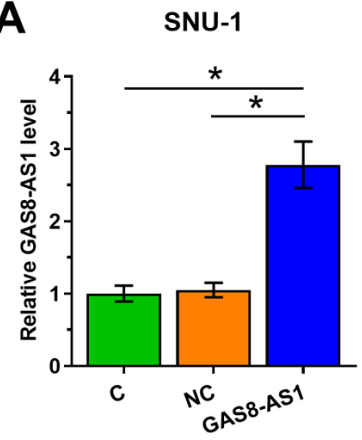

C

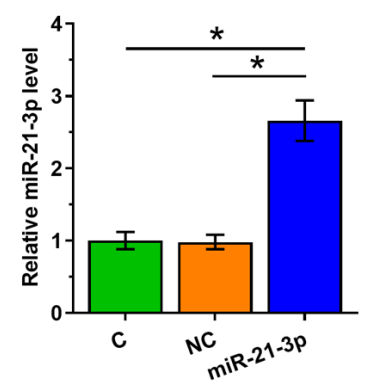

E

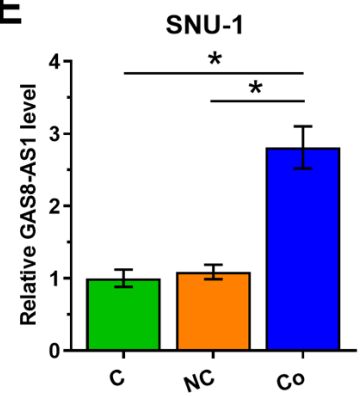

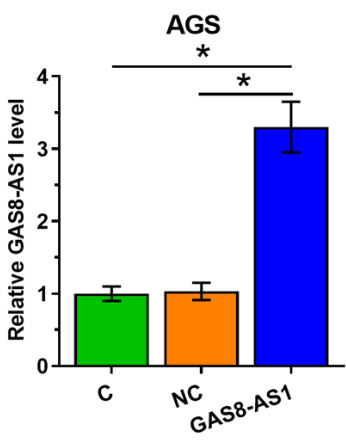

AGS
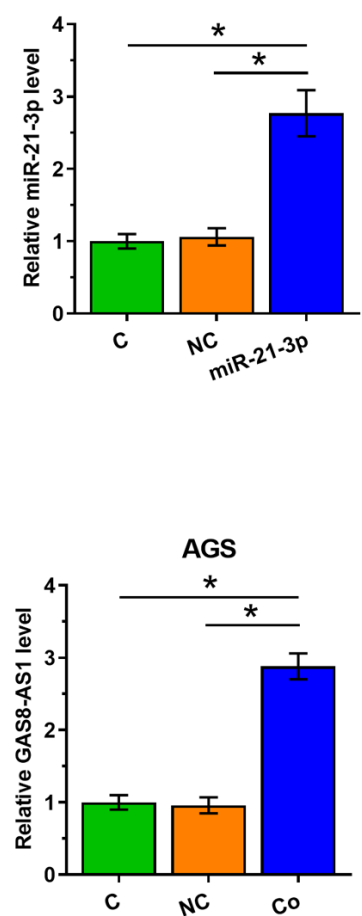

B

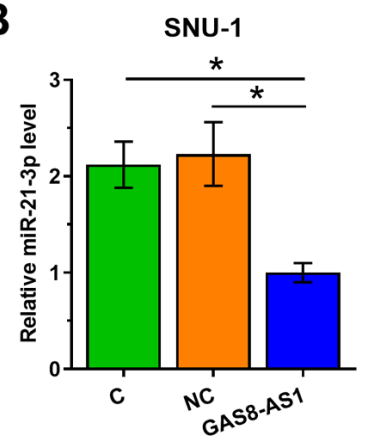

D

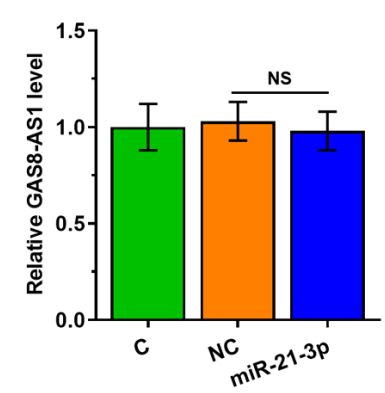

$\mathbf{F}$

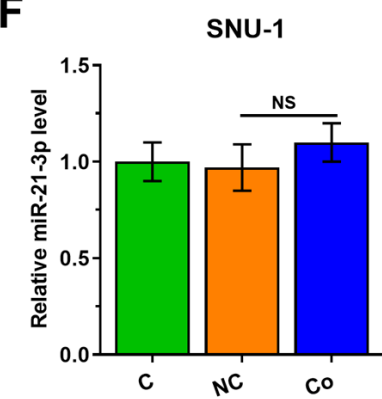

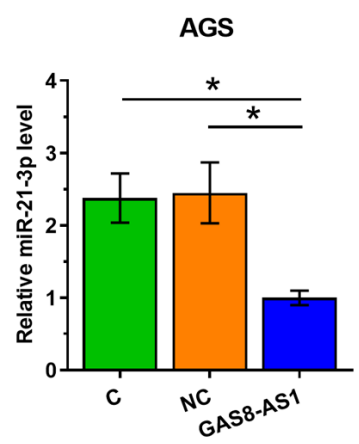

AGS

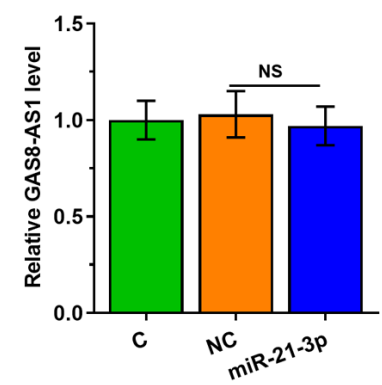

AGS

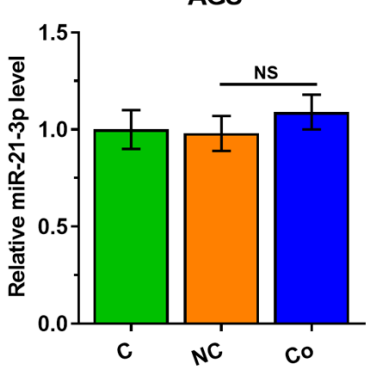

Figure 5. GAS8-AS1 is an upstream inhibitor of miR-21-3p in gastric cancer cells. (A) GAS8-AS1 was overexpressed in SNU-1 and AGS cells at $24 \mathrm{~h}$ after transfection. (B) Overexpression of GAS8-AS1 decreased the expression levels of miR-21-3p. (C) miR-21-3p was overexpressed in SNU-1 and AGS cells $24 \mathrm{~h}$ after transfection. (D) Overexpression of miR-21-3p did not alter the expression of GAS8-AS1. Co-transfection of GAS8-AS1 expression vector and miR-21-3p mimic led to (E) upregulated GAS8-AS1, but (F) did not affect the expression of miR-21-3p. " $\mathrm{P}<0.05$. Co, co-transfection; miR, microRNA, C, control; NC, negative control; NS, not significant. 
A

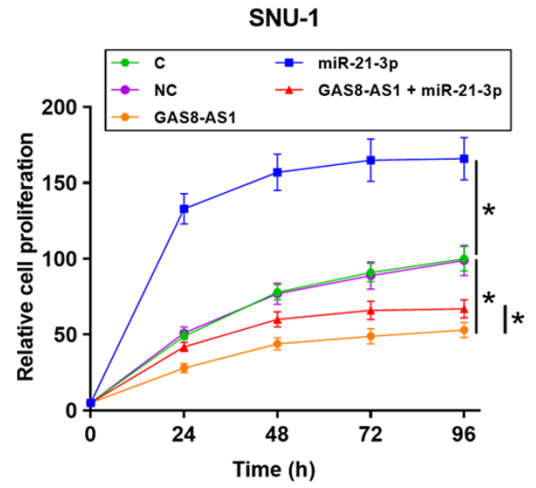

B

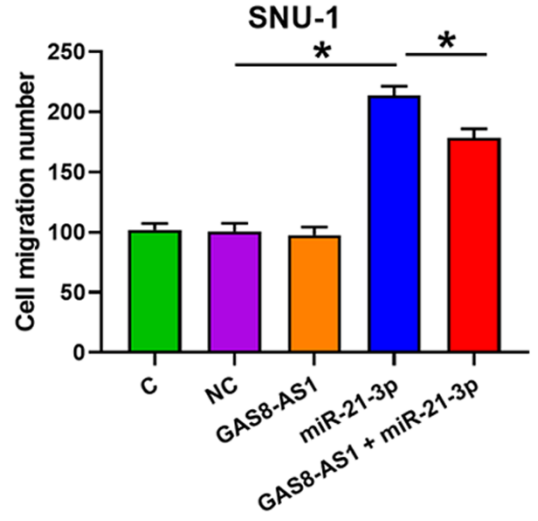

C

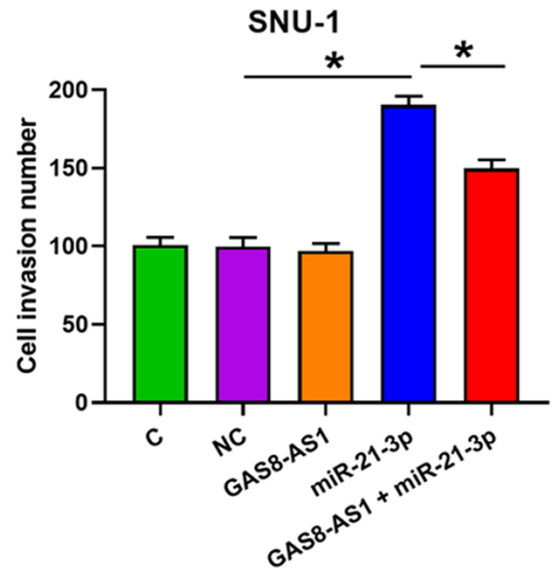

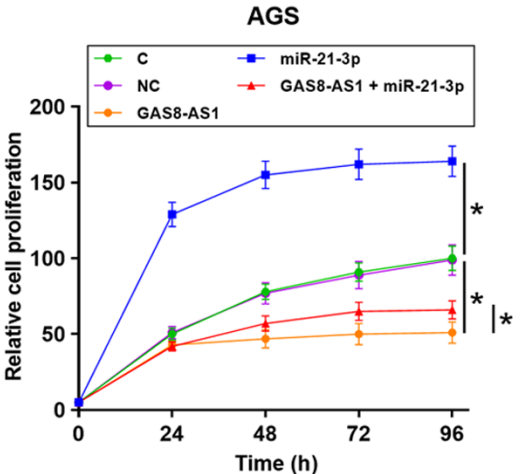

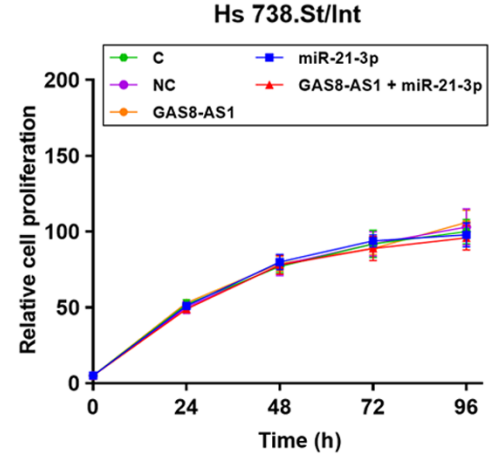

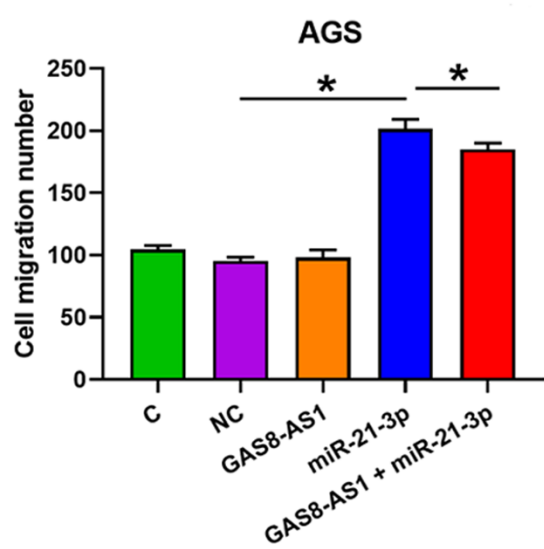

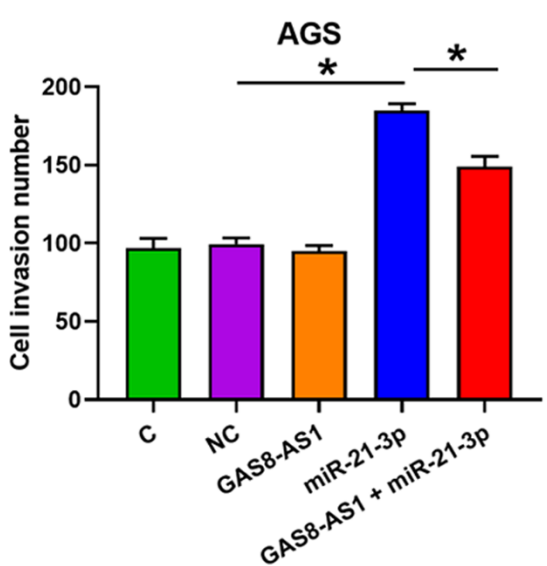

Figure 6. GAS8-AS1 regulates cancer cell proliferation through miR-21-3p. (A) Overexpression of GAS8-AS1 inhibited cancer cell proliferation, while overexpression of miR-21-3p promoted cancer cell proliferation and attenuated the effects of GAS8-AS1 overexpression. In addition, overexpression of GAS8-AS1 and miR-21-3p did not affect the proliferation of normal Hs 738.St/Int cells. (B) Overexpression of miR-21-3p promoted cancer cell migration, while overexpression of GAS8-AS1 did not affect cell migration. (C) Overexpression of miR-21-3p promoted cancer cell invasion, while overexpression of GAS8-AS1 did not affect cell invasion. ${ }^{*} \mathrm{P}<0.05$. miR, microRNA; C, control; NC, negative control.

As shown in Fig. 6A, the overexpression of GAS8-AS1 significantly inhibited cancer cell proliferation, while the overexpression of miR-21-3p inhibited cancer cell proliferation $(\mathrm{P}<0.05)$. In addition, the cells transfected with GAS8-AS1 and miR-21-3p had enhanced cell proliferation compared with the cells transfected with the GAS8-AS1 expression vector. In addition, the overexpression of GAS8-AS1 and miR-21-3p did not affect the proliferation of the normal Hs 738.St/Int cell line. Furthermore, the overexpression of miR-21-3p markedly enhanced cancer cell migration and invasion compared with the NC group, while the overexpression of GAS8-AS1 did not affect the migration and invasion of gastric cancer cells compared with the NC group (Figs. 6B and C, and S1; P<0.05). However, the overexpression of GAS8-AS1 partly alleviated the effect of 
miR-21-3p overexpression on cancer cell migration and invasion compared with the cells transfected with miR-21-3p mimic.

\section{Discussion}

The survival rate of patients with gastric cancer currently remains poor, particularly for patients at stages III and IV (25). Therefore, accurate prognosis is critical for the development of effective treatments. A considerable number of prognostic biomarkers have been identified for gastric cancer. For example, lncRNA MALAT1 has been found to be significantly upregulated in gastric cancer and serves as a predictor of poor survival (26). Furthermore, lncRNA LINC-PINT was demonstrated to be downregulated in patients with gastric cancer and predicted poor survival (27). In the present study, downregulation of GAS8-AS1 in patients with gastric cancer was observed. In addition, low expression levels of GAS8-AS1 predicted poor survival of patients with gastric cancer compared with high expression levels. Therefore, GAS8-AS1 may serve as a potential prognostic marker for gastric cancer. Of note is that no significant difference was found in the expression levels of GAS8-AS1 among the four clinical stages.

Studies have revealed that GAS8-AS1 is a tumor suppressor in papillary thyroid carcinoma, osteosarcoma and glioblastoma (14-16). In the present study, it was revealed that the overexpression of GAS8-AS1 significantly inhibited gastric cancer cell proliferation compared with the $\mathrm{NC}$, suggesting an anticancer role of GAS8-AS1 in gastric cancer. To the best of our knowledge, the present study was the first to report the role of GAS8-AS1 in gastric cancer.

miR-21-3p has been identified as a critical regulator in gastric cancer (20). In the present study, miR-21-3p expression was markedly upregulated in the gastric cancer tissues compared with non-cancerous tissues, and its expression was negatively correlated with the expression of GAS8-AS1 in gastric cancer tissues. In addition, miR-21-3p was predicted to be a potential target of GAS8-AS1, and this interaction was confirmed using an RNA pull-down assay. Moreover, it was observed that GAS8-AS1 inhibited the expression of miR-21-3p, while overexpression of miR-21-3p exhibited no effect on the expression level of GAS8-AS1. Therefore, we hypothesized that GAS8-AS1 may inhibit the expression of miR-21-3p by directly sponging it. To the best of our knowledge, this is the first study to report the association between GAS8-AS1 and miR-21-3p. In addition, overexpression of miR-21-3p promoted cancer cell proliferation compared with the $\mathrm{NC}$, and alleviated GAS8-AS1-mediated inhibition of cancer cell proliferation in two cancer cell lines compared with that in GAS8-AS1 overexpression group. Collectively, the results of the present study indicate that GAS8-AS1 may play a protective role in gastric cancer by regulating miR-21-3p.

However, the present study has some limitations. Firstly, the sample size of patients with gastric cancer was small. Future studies will collect more samples to strengthen the findings. In addition, in vivo experiments were not performed in the study, but will be performed in future studies to further confirm the role of GAS8-AS1 in gastric cancer.

In conclusion, GAS8-AS1 was found to be significantly downregulated in cancer tissues compared with non-cancerous tissues in patients with gastric cancer, and low expression levels of GAS8-AS1 predicted poor survival. Furthermore, the results showed that GAS8-AS1 may inhibit gastric cancer cell proliferation by downregulating miR-21-3p. Future experiments will focus on the detailed mechanisms by which GAS8-AS1 exerts its anticancer function in gastric cancer.

\section{Acknowledgements}

Not applicable.

\section{Funding}

No funding was received.

\section{Availability of data and materials}

The datasets used and/or analyzed during the current study are available from the corresponding author on reasonable request.

\section{Authors' contributions}

CL was responsible for writing the manuscript, performing the literature search, data analysis and statistical analysis. HW conducted the data and statistical analyses. SM, JH, LY and YS performed the literature search, and were responsible for data collection and sample collection. XZ was responsible for designing the study and revising the manuscript. CL and XZ confirm the authenticity of all the raw data. All authors have read and approved the final manuscript.

\section{Ethics approval and consent to participate}

The present study received ethics approval from the Ethics Committee of the Affiliated Hospital of Xuzhou Medical University (Xuzhou, China) prior to patient admission. Written informed consent was obtained from all individual participants included in this study.

\section{Patient consent for publication}

Not applicable.

\section{Competing interests}

The authors declare that they have no competing interests.

\section{References}

1. Rawla P and Barsouk A: Epidemiology of gastric cancer: Global trends, risk factors and prevention. Prz Gastroenterol 14: 26-38, 2019.

2. Sumiyama K: Past and current trends in endoscopic diagnosis for early stage gastric cancer in Japan. Gastric Cancer 20 (Suppl 1): 20-27, 2017.

3. Li X, Zhang Y, Zhang H, Liu X, Gong T, Li M, Sun L, Ji G, Shi Y, Han Z, et al: miRNA-223 promotes gastric cancer invasion and metastasis by targeting tumor suppressor EPB41L3. Mol Cancer Res 9: 824-833, 2011 .

4. Wang CJ, Zhu CC, Xu J, Wang M, Zhao WY, Liu Q, Zhao G and Zhang ZZ: The IncRNA UCA1 promotes proliferation, migration, immune escape and inhibits apoptosis in gastric cancer by sponging anti-tumor miRNAs. Mol Cancer 18: 115, 2019

5. Hu X, Zhang M, Miao J, Wang X and Huang C: miRNA-4317 suppresses human gastric cancer cell proliferation by targeting ZNF322. Cell Biol Int 42: 923-930, 2018. 
6. Wei GH and Wang X: lncRNA MEG3 inhibit proliferation and metastasis of gastric cancer via p53 signaling pathway. Eur Rev Med Pharmacol Sci 21: 3850-3856, 2017.

7. Shao Y, Li J, Lu R, Li T, Yang Y, Xiao B and Guo J: Global circular RNA expression profile of human gastric cancer and its clinical significance. Cancer Med 6: 1173-1180, 2017.

8. Poursheikhani A, Abbaszadegan MR, Nokhandani N and Kerachian MA: Integration analysis of long non-coding RNA (lncRNA) role in tumorigenesis of colon adenocarcinoma BMC Med Genomics 13: 108, 2020.

9. Ma Z, Gu G, Pan W and Chen X: Chen X and therapy: LncRNA PCAT6 accelerates the progression and chemoresistance of cervical cancer through up-regulating ZEB1 by sponging miR-543. OncoTargets Ther 13: 1159-1170, 2020

10. Zhang L, Wang L, Wang Y, Chen T, Liu R, Yang W, Liu Q and Tu K: LncRNA KTN1-AS1 promotes tumor growth of hepatocellular carcinoma by targeting miR-23c/ERBB2IP axis. Biomed Pharmacother 109: 1140-1147, 2019.

11. Sun M, Nie F, Wang Y, Zhang Z, Hou J, He D, Xie M, Xu L, De W, Wang Z, et al:: LncRNA HOXA11-AS Promotes Proliferation and Invasion of Gastric Cancer by Scaffolding the Chromatin Modification Factors PRC2, LSD1, and DNMT1. Cancer Res 76: 6299-6310, 2016

12. Li H, Yu B, Li J, Su L, Yan M, Zhu Z and Liu B: Overexpression of lncRNA H19 enhances carcinogenesis and metastasis of gastric cancer. Oncotarget 5: 2318-2329, 2014.

13. Li H and Wang Y: Long Noncoding RNA (lncRNA) MIR22HG Suppresses Gastric Cancer Progression through Attenuating NOTCH2 Signaling. Med Sci Monit 25: 656-665, 2019.

14. Chen N, Yin D, Lun B and Guo X: LncRNA GAS8-AS1 suppresses papillary thyroid carcinoma cell growth through the miR-135b-5p/CCND2 axis. Biosci Rep 9: BSR20181440, 2019.

15. Zha Z, Han Q, Liu W and Huo S: IncRNA GAS8-AS1 downregulates lncRNA UCA1 to inhibit osteosarcoma cell migration and invasion. J Orthop Surg Res 15: 38, 2020.

16. Wu X, Jiang T, Huang R and Xiao X: LncRNA GAS8-AS1 downregulates lncRNA NEAT1 to inhibit glioblastoma cell proliferation. Brain Behav 11: e02128, 2021.

17. Qin Y, Sun W, Wang Z, Dong W, He L, Zhang T, Shao L and Zhang H: ATF2-Induced IncRNA GAS8-AS1 Promotes Autophagy of Thyroid Cancer Cells by Targeting the miR-187-3p/ATG5 and miR-1343-3p/ATG7 Axes. Mol Ther Nucleic Acids 22: 584-600, 2020.
18. Wu Y, Song Y, Xiong Y, Wang X, Xu K, Han B, Bai Y, Li L, Zhang Y, Zhou L: MicroRNA-21 (Mir-21) Promotes Cell Growth and Invasion by Repressing Tumor Suppressor PTEN in Colorectal Cancer. Cell Physiol Biochem 43: 945-958.

19. Wang H, Tan Z, Hu H, Liu H, Wu T, Zheng C, Wang X, Luo Z, Wang J, Liu S, et al: microRNA-21 promotes breast cancer proliferation and metastasis by targeting LZTFL1. BMC Cancer 19: 738, 2019.

20. Li Q, Li B, Li Q, Wei S, He Z, Huang X, Wang L, Xia Y, Xu Z, $\mathrm{Li} \mathrm{Z}$, et al: Exosomal miR-21-5p derived from gastric cancer promotes peritoneal metastasis via mesothelial-to-mesenchymal transition. Cell Death Dis 9: 854, 2018.

21. Yan J, Liu T, Zhou X, Dang Y, Yin C and Zhang G: FZD6, targeted by miR-21, represses gastric cancer cell proliferation and migration via activating non-canonical wnt pathway. Am J Transl Res 8: 2354-2364, 2016.

22. Amin MB, Edge SB, Greene FL, Byrd DR, Brookland RK, Washington MK, Gershenwald JE, Compton CC, Hess KR, Sullivan DC, et al (Eds.). In: AJCC Cancer Staging Manual, 8th edition. Springer, Cham, Switzerland, pp 3077-3079, 2017.

23. Livak KJ and Schmittgen TD: Analysis of relative gene expression data using real-time quantitative PCR and the $2(-\Delta \Delta$ C(T)) Method. Methods 25: 402-408, 2001.

24. Fluss R, Faraggi D and Reiser B: Estimation of the Youden Index and its associated cutoff point. Biom J 47: 458-472, 2005.

25. Song Z, Wu Y, Yang J, Yang D and Fang X: Progress in the treatment of advanced gastric cancer. Tumour Biol 39: 1010428317714626, 2017

26. Dai Q, Zhang T and Li C: LncRNA MALAT1 regulates the cell proliferation and cisplatin resistance in gastric cancer via PI3K/AKT pathway. Cancer Manag Res 12: 1929-1939, 2020.

27. Feng $\mathrm{H}$, Zhang J, Shi Y, Wang L, Zhang $\mathrm{C}$ and Wu L: Long noncoding RNA LINC-PINT is inhibited in gastric cancer and predicts poor survival. J Cell Biochem 120: 9594-9600, 2019. 